\title{
UV-Spectroscopic Detection of (Pyro-)Phosphate with the PUB module
}

\author{
Lea Eilert, ${ }^{1}$ Anett Schallmey, ${ }^{1}$ and Felix Kaspar, ${ }^{1,2 *}$ \\ 1 Technische Universität Braunschweig, Institute for Biochemistry, Biotechnology and Bioinformatics, \\ Spielmannstraße 7, 38106, Braunschweig, Germany. \\ 2 Chair of Bioprocess Engineering, Institute of Biotechnology, Faculty III Process Sciences, Technische \\ Universität Berlin, Straße des 17. Juni 135, D-10623, Berlin, Germany.
}

Despite the prevalence of ortho- and pyrophosphate in biochemistry, operationally simple and versatile high-throughput
methodologies for their quantification are lacking. We herein introduce PUB, a module for phosphate detection by continuous
UV-spectroscopic monitoring of 5 -bromouridine phosphorolysis. The PUB module employs cheaply available, bench-stable
reagents and can be employed for continuous and discontinuous reaction monitoring in biochemical assays to detect
(pyro-)phosphate concentrations spanning almost four orders of magnitude, as demonstrated with representative use-cases.

Phosphate groups are ubiquitous across the biological sciences as they serve as allosteric modifiers, solubility anchors or high-energy leaving groups (Figure 1a). ${ }^{[1]}$ As such, nature has developed a myriad of enzymes to shuffle phosphate groups between substrates and/or utilize energyrich phosphorylated cosubstrates to power otherwise unfavorable reactions (Fig. 1b). Examples of such enzymes span transferases, ligases and lyases and account for more than 10 million entries in the Uniprot database. Despite their prevalence, the experimental interrogation of many of these enzyme functions (particularly their kinetics) remains difficult since the detection of inorganic (pyro-)phosphate is not trivial in many biochemical assays. Notable previous developments toward this end include fluorescent phosphate binding proteins, ${ }^{[2]}$ fluorescent ionic dyes with tunable photochemical properties, ${ }^{[3]}$ chromogenic metal complexes ${ }^{[4]}$ and coupled enzymatic assays employing purine nucleoside phosphorylases and xanthine-oxidases to generate a UV signal. ${ }^{[5]}$ However, these methods typically only allow for the examination of a small experimental space, exhibit low selectivity, or employ expensive reagents unsuited for highthroughput experimentation (see the Supplementary Information and Table S1 for a detailed literature analysis). Thus, cheaply available, operationally simple, and versatile methods, which are compatible with biochemical assays and high-throughput experimentation, are lacking. To close this gap, we developed PUB, a compact module for phosphate detection via UV V-spectroscopic monitoring of 5-bromouridine phosphorolysis. Herein, we introduce the PUB module, outline its application to biochemical assays on various scales and illustrate its utility with selected use-cases.

The PUB module relies on a highly selective nucleoside phosphorolysis step, which can be leveraged to generate a UV spectroscopic read-out in the presence of phosphate. Given the inherent limitations of existing purine-based systems, which employ irreversible enzymatic phosphorolysis of a nucleoside precursor, we recognized that a conceptual redesign would be necessary to realize the versatility and scope required for many applications. Based on our previous forays into nucleoside phosphorylase chemistry $^{[6-9]}$ and UV spectroscopic reaction monitoring, ${ }^{[10,11]}$ we hypothesized that we could employ in situ deprotonation of a pyrimidine nucleoside and its corresponding nucleobase, which then tautomerizes to a pyrimidinolate, to yield a spectral redshift (Fig. 1c). Thus, we envisioned that the phosphorolysis of a pyrimidine nucleoside could report on the presence of phosphate in solution through a characteristic UV spectroscopic signal reflecting conversion of the starting material. A systematic examination of commercially available uridine analogs identified 5-bromouridine (1a) as the ideal candidate. The nucleoside 1a ( $\left.p K_{a}=7.95\right)$ can be deprotonated under mildly alkaline conditions in buffered solution and its corresponding anionic nucleobase $\mathbf{1 b}\left(p K_{a}=8.03\right)$ exhibits a $22 \mathrm{~nm}$ UV redshift, enabling their spectroscopic differentiation. Monitoring of the phosphorolysis of $\mathbf{1 a}$ to $1 \mathbf{b}$ at $\mathrm{pH} 9$ yields highly overlapping absorption spectra with an isosbestic point at $288 \mathrm{~nm}$ (Fig. 1d) and a maximum difference of the extinction coefficients at $304 \mathrm{~nm}\left(\Delta \varepsilon_{304}=5.4 \mathrm{mM}^{-1} \mathrm{~cm}^{-1}\right.$, Figs. 1e and 1f). Thus, monitoring at $304 \mathrm{~nm}$ coupled with isosbestic point normalization via parallel monitoring at $288 \mathrm{~nm}$ provides quantitative real-time conversion data for this transformation, given reference values for both compounds (Fig. 1g). As initial studies indicated that this reaction could be carried out with a variety of pyrimidine nucleoside phosphorylases, we elected the commercially available thermostable variant Y02 for further studies. An extensive evaluation of buffer systems revealed that the PUB module performs best in amino acid buffers such as asparagine $\left(p K_{a}=8.8\right)$ or glycine $\left(p K_{a}=9.6\right)$ or the amino sulfonate taurine $\left(p K_{a}=9.1\right)$. Sulfonic acids from Good's series $^{[12]}$ and the "better buffer" diethylpiperazine ${ }^{[13]}$ gave inferior results while other buffers including ammonia, borate or carbonate proved incompatible (see the SI for a detailed analysis and buffer recommendations; also see the SI section "Additional considerations"). Owing to the inherent selectivity of nucleoside phosphorlyases, the PUB module is highly selective to inorganic phosphate and exhibits no cross-reactivity with other phosphate species. Similarly, we found that the presence of other ions in solution has no significant impact on the performance of the module.

The optimized PUB module is a highly robust system capable of detecting (pyro-)phosphate over a remarkable concentration range by exploiting principles of thermodynamic control. While previous nucleoside phosphorylase-based systems have used irreversible phosphorolytic steps (either via product removal or by rendering the reverse direction kinetically unfeasible), the PUB module employs a reversible phosphorolysis, which yields a significantly simplified system in terms of required materials. As such, the PUB module utilizes the tight thermodynamic control of the phosphorolysis of 1a $(K=$ $0.16)^{[8]}$ to detect multiple equivalents of phosphate per nucleoside (Fig. 1h). Using modifications of previously published equations, ${ }^{[7]}$ the extent of conversion in an equilibrium state of this transformation (as monitored spectroscopically via the $\mathrm{A}_{304} / \mathrm{A}_{288}$ ratio) provides the phosphate concentration in solution with high accuracy and reproducibility. In practice, this means that $100 \mu \mathrm{M}$ to $>5 \mathrm{mM}$ phosphate can readily be detected with standard spectro- 
The versatility of phosphate in nature is driven by a vast array of phosphate-shuffling enzymes<smiles>COC(=O)CN(C)C(=N)NP(=O)([O-])[O-]</smiles>

energy storage

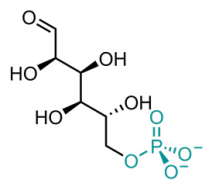

ionization anchor<smiles>C=C(O)C(=O)OC1CC(C(=O)O)C(O)CC1C(=O)O</smiles>

leaving group

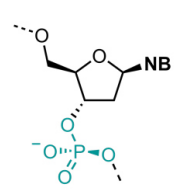

solubility anchor

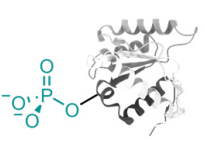

allosteric regulator

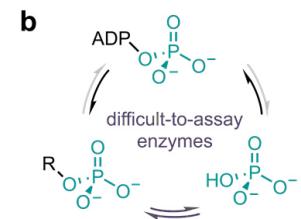

The PUB module enables quantitative phosphate detection in biochemical assays

c

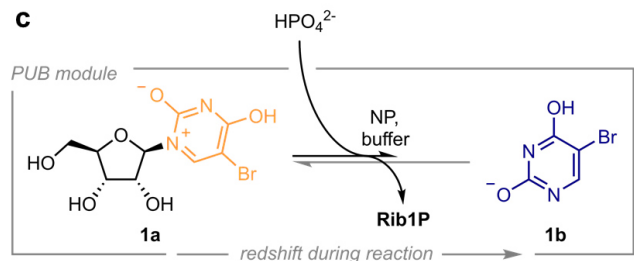

g

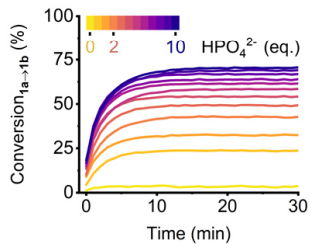

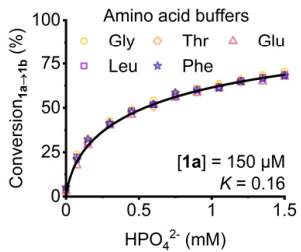

d
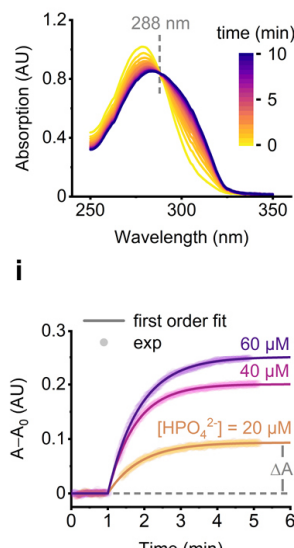

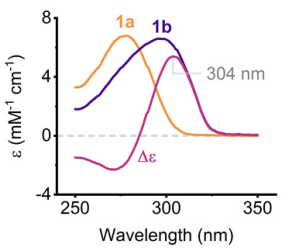

j

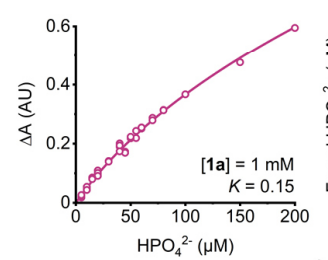

f

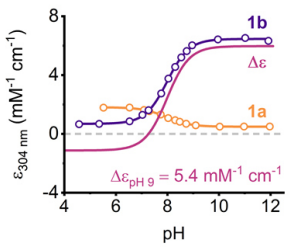

$\mathbf{k}$

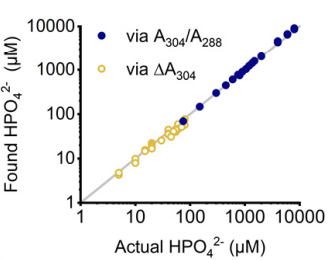

— High phosphate concentrations via 304/288 ratio and thermodynamic control $\_$- Low phosphate concentrations via $\triangle A$ and thermodynamic control (phosphate in excess over 1a)

Coupling of PUB to an inorganic pyrophosphatase enables quantitative detection of pyrophosphate

I

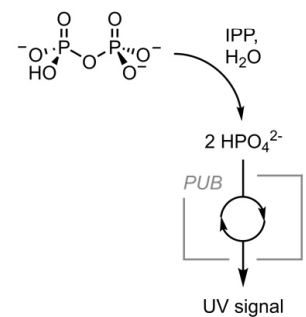

m

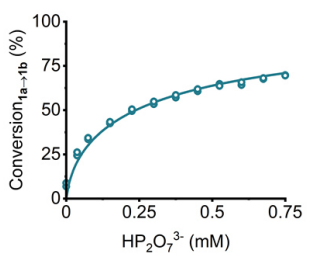

n

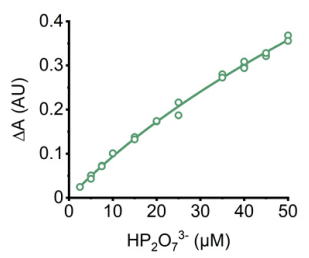

o

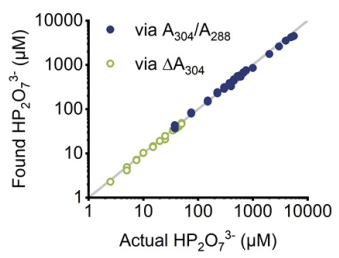

PUB facilitates biochemical studies, e.g. enzyme characterization

p

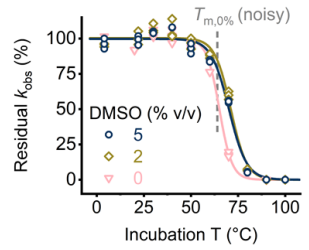

$\mathbf{q}$

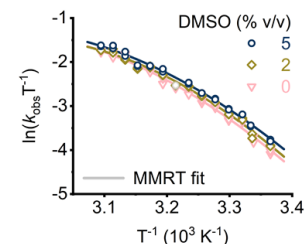

$\mathbf{r}$

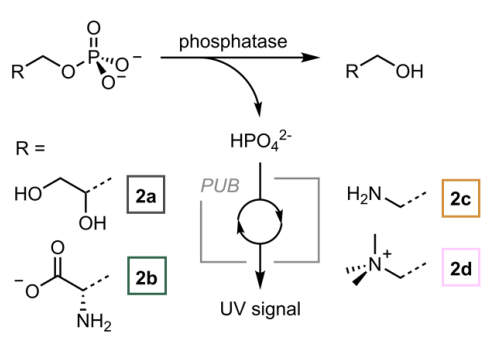

S

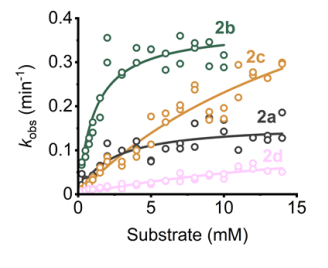

Figure 1. a Examples of phosphorylated biomolecules, b Phosphate turnover in vivo requires enzyme for phosphate shuffling, c The PUB module employs pyrimidine nucleoside phosphorolysis under alkaline conditions, d UV absorption spectra obtained during phosphorolysis of $\mathbf{1 a}$ at $\mathrm{pH}$, e Spectroscopic properties of $\mathbf{1 a}$ and $\mathbf{1 b}$ at $\mathrm{pH} \mathbf{9}, \mathbf{f} \mathrm{pH}$-dependence of the spectroscopic properties, $g$ Continuous reaction monitoring of the $\mathbf{1 a} \rightarrow \mathbf{1 b}$ transformation via isosbestic point normalization, $h$ Thermodynamic control of the phosphorolysis of 1a enables the detection of multiple equivalents of phosphate in various amino acid buffer systems, i Extinction differences enable detection of low phosphate concentrations, $\mathbf{j}$ Non-linear response of $\Delta \mathrm{A}$ to phosphate due to thermodynamic control, $\mathbf{k}$ Phosphate can be detected accurately over concentrations spanning more than three orders of magnitude, I Enzymatic hydrolysis of pyrophosphate to orthophosphate, $\mathbf{m}$-o Detection of pyrophosphate via thermodynamic control following hydrolysis, $\mathbf{p}$ Thermal stability of the IPP from Geobacillus stearothermophilus, $\mathbf{q}$ Eyring plots of IPP-catalyzed pyrophosphate hydrolysis, revealing curvature due to an activation heat capacity change, $\mathbf{r}$ Phosphatase-catalyzed hydrolysis of organophosphates, s Michaelis-Menten kinetics with selected model substrates obtained via high-throughput experimentation. $\mathrm{PUB}=$ phosphate detection via UV spectroscopic monitoring of 5-bromouridine phosphorolysis, NB = nucleobase, $\mathrm{NP}=$ nucleoside phosphorylase, Rib1P = ribose-1-phosphate, eq. = equivalent, IPP = inorganic pyrophosphatase, DMSO = dimethylsulfoxide, $K=$ equilibrium constant. All data are freely available online. ${ }^{[14]}$ 
scopic equipment, with continuous and discontinuous monitoring approaches giving essentially identical results in a high-throughput setup. Notably, this approach can be carried out in $1 \mathrm{~mL}$ cuvettes in a photometer, $200 \mu \mathrm{L}$ multiwell plates in a plate reader or even in $1 \mu \mathrm{L}$ droplets assessed with a nanophotometer. Lower phosphate concentrations down to around $2 \mu \mathrm{M}$ can be detected by providing 1a in excess and measuring absorbance differences between a baseline and a near-equilibrium state (Figs. $1 \mathrm{i}$ and $1 \mathrm{j}$ ). The latter can be fitted with first-order kinetic assumptions for an accurate assessment of phosphate content up to at least $200 \mu \mathrm{M}$ (see the SI for details, equations and workflow). Collectively, this enables the quantification of phosphate over a concentration range spanning nearly four orders of magnitude (Fig. 1k). While we recommend analysis at $\mathrm{pH} 9$ for optimal performance, the PUB module can also be employed at lower $\mathrm{pH}$ values, albeit with reduced sensitivity due to smaller $\Delta \varepsilon$ values (Fig. 1f; please also see the SI section "Additional considerations"). The extension of this methodology by an inorganic pyrophosphatase (IPP, hydrolyzing pyrophosphate to orthophosphate, Fig. 1I) enables the quantification of pyrophosphate in a fully analogous fashion, employing identical reaction conditions (Figs. $1 \mathrm{~m}-\mathrm{o}$ ).

As all these approaches are readily compatible with continuous reaction monitoring and high-throughput experimentation, the PUB module is a remarkably useful tool for kinetic studies and enzyme characterization, as we demonstrated with selected use-cases. For instance, we assessed the thermal stability of the IPP from Geobacillus stearothermophilus and found the $T_{50}^{10}$ value determined through kinetic experiments to be in excellent agreement with the noisy $T_{m}$ obtained via differential scanning fluorimetry (Fig. $1 \mathrm{p}$ ). As the $\Delta \varepsilon_{304}$ between $\mathbf{1 a}$ and $\mathbf{1 b}$ is not temperature-dependent and $\mathrm{Y} 02$ readily allows experimentation up to at least $60^{\circ} \mathrm{C}$ (see the SI for details), we used the PUB module to obtain Eyring plots for the IPPcatalyzed pyrophosphate hydrolysis (Fig. 1q). This analysis revealed the presence of a moderate activation heat capacity change $\Delta C_{p}^{*}$ across different conditions, ${ }^{[9,15]}$ which would have been impossible or extremely laborious to examine with existing phosphate detection methods. In a similar manner, the PUB module allowed the straightforward characterization of an alkaline phosphatase with a series of model substrates $\mathbf{2 a - 2 d}$ (Figs. $1 \mathbf{r}$ and $1 \mathrm{~s}$ ). Notably, these experiments could routinely be performed in a multi-well format running more than 30 reactions in parallel.

In conclusion, we have developed PUB, an operationally simple module for the detection of inorganic (pyro-)phosphate in biochemical assays. This method is cheap, versatile, and readily compatible with standard spectroscopic equipment and high-throughput experimentation on various scales.

\section{Acknowledgements}

The authors thank BioNukleo $\mathrm{GmbH}$ for generously supporting this research with thermostable enzymes and the Institute of biochemical engineering (ibvt, TU Braunschweig) for further support. In addition, the authors are grateful to the members of the Schallmey group, Dr. M. Rhia L. Stone (Rutgers University), Dr. Anke Kurreck (BioNukleo $\mathrm{GmbH}$ ), and Kerstin Heinecke (Physikalisch-Technische Bundesanstalt) for proofreading and comments on this manuscript and its extensive supplementary information.

\section{Conflict of Interest}

This research was supported in part by BioNukleo $\mathrm{GmbH}$, a biotech company selling nucleoside analogues and nucleoside phosphorylases.

\author{
Author Information \\ Corresponding Author \\ Dr. Felix Kaspar \\ felix.kaspar@web.de / felix.kaspar@tu-bs.de, \\ orcid.org/0000-0001-6391-043X
}

Other Authors

Lea Eilert, orcid.org/0000-0002-4753-1322

Dr. Anett Schallmey, orcid.org/0000-0002-6670-0574

\section{Supplementary Information}

The following references appear in the Supplementary Information:

CRediT system ${ }^{[16]}$

Scientific color maps ${ }^{[17]}$

Golden Gate cloning ${ }^{[18]}$

Phosphate detection from plant samples ${ }^{[19]}$

Phosphate detection methods

Malachite green-based assays ${ }^{[20-24]}$

Other molybdate-based assays ${ }^{[25-35]}$

Coumarin-based sensors ${ }^{[36]}$

NADH-based enzymatic assays ${ }^{[37-45]}$

Xanthine oxidase-based enzymatic assays ${ }^{[46-54]}$

Methyl-purine-based enzymatic assays ${ }^{[55-58]}$

Thymidine phosphorylase-based, HPLC-coupled assays $^{[59]}$

Maltose phosphorylase-based assays ${ }^{[60]}$

Pyruvate oxidase-based assays[61]

Phosphate-binding proteins ${ }^{[62-65]}$

Metal-binding fluorescent dyes ${ }^{[66]}$

Colorimetric assays with insolubles ${ }^{[67,68]}$

Capillary electrophoresis ${ }^{[69]}$

Pyrophosphate detection methods

NMR-based detection ${ }^{[70]}$

$\mathrm{Zn}^{2+}$-based fluorescent sensors ${ }^{[71-102]}$

$\mathrm{Cu}^{2+}$-based fluorescent sensors ${ }^{[103-110]}$

$\mathrm{Cd}^{2+}$-based fluorescent sensors ${ }^{[111,112]}$

$\mathrm{Ga}^{3+}$-based fluorescent sensors ${ }^{[113]}$

$\mathrm{Fe}^{3+}$-based fluorescent sensors ${ }^{[14-116]}$

$\mathrm{Al}^{3+}$-based fluorescent sensors ${ }^{[117-119]}$

Amide-based sensors ${ }^{[104]}$

Amine-based sensors ${ }^{[120]}$

Carboxylate-based sensors ${ }^{[121]}$

Imidazolium-based sensors ${ }^{[122-124]}$

Pyridinium-based sensors ${ }^{[125-127]}$

Pyrrole-based sensors ${ }^{[128-130]}$

Guanidinium-based sensors ${ }^{[131]}$

(Thio-)Urea-based sensors ${ }^{[132-135]}$

Gold nanoclusters ${ }^{[136]}$

Xanthine oxidase-based enzymatic assays ${ }^{[137]}$

NADH-based enzymatic assays ${ }^{[138]}$

UDP-glucose phosphorylase-based enzymatic assays ${ }^{[139,140]}$

Luciferase-based assays ${ }^{[141-146]}$

Electrochemical methods ${ }^{[147]}$

Precipitation ${ }^{[148]}$

Heterologous xanthine oxidase expression [149-151]

Xanthine oxidase instability ${ }^{[152-154]}$

Instability of 7-methylated purine nucleosides ${ }^{[155]}$

Biocatalytic nucleoside synthesis ${ }^{[156,157]}$

Thermostable nucleoside phosphorylases ${ }^{[158-164]}$

5-Fluorouracil $p K_{a}{ }^{[165]}$

$p K_{a}$ calculation $^{[166]}$

Nucleoside phosphorolysis reaction monitoring ${ }^{[167]}$ 
Ammonia vapor pressure ${ }^{[168]}$

Potential carbonate binding to phosphorylases ${ }^{[169]}$

Pyrophosphate hydrolysis thermodynamics ${ }^{[170]}$

GstIPP[171]

Solubility of magnesium pyrophosphates ${ }^{[172]}$

Differential scanning fluorimetry ${ }^{[173]}$

Protparam ${ }^{[174]}$

MMRT model ${ }^{[175-177]}$

Transition state theory ${ }^{[178]}$

Akaike's Information Criterion [179]

Enzymes with non-zero $\Delta C_{p}^{*}[176,180-189]$

Inflection point hypothesis ${ }^{[190]}$

Sugar phosphate stability ${ }^{[191]}$

Arsenolysis ${ }^{[192]}$

\section{References}

[1] H. F. Westheimer, Science 1987, 235, 1173-1178.

[2] M. Brune, J. L. Hunter, J. E. T. Corrie, M. R. Webb, Biochemistry 1994, 33, 8262-8271.

[3] J. Fischbach, Q. Loh, F. F. Bier, T. S. Lim, M. Frohme, J. Glökler, Sci. Rep. 2017, 7, 45085.

[4] P. A. Lanzetta, L. J. Alvarez, P. S. Reinach, O. A Candia, Anal. Biochem. 1979, 100, 95-97.

[5] H. de Groot, H. de Groot, T. Noll, Biochem. J. 1985, 230, 255-260.

[6] F. Kaspar, R. T. Giessmann, K. F. Hellendahl, P. Neubauer, A. Wagner, M. Gimpel, ChemBioChem 2020, 21, 1428-1432.

[7] F. Kaspar, M. Seeger, S. Westarp, C. Köllmann, A Lehmann, P. Pausch, S. Kemper, P. Neubauer, G. Bange, A. Schallmey, et al., ACS Catal. 2021, 11, 10830-10835.

[8] F. Kaspar, R. T. Giessmann, P. Neubauer, A. Wagner, M. Gimpel, Adv. Synth. Catal. 2020, 362, 867-876.

[9] F. Kaspar, D. S. Wolff, P. Neubauer, A. Kurreck, V. L. Arcus, Biochemistry 2021, 60, 1573-1577.

[10] F. Kaspar, R. T. Giessmann, N. Krausch, P. Neubauer, A. Wagner, M. Gimpel, Methods Protoc. 2019, 2, 60.

[11] F. Kaspar, R. T. Giessmann, S. Westarp, K. F. Hellendahl, N. Krausch, I. Thiele, M. C. Walczak, P. Neubauer, A. Wagner, ChemBioChem 2020, 21, 2604

[12] N. E. Good, G. D. Winget, W. Winter, T. N. Connolly, S. Izawa, R. M. M. Singh, Biochemistry 1966, 5, 467-477.

[13] A. Kandegedara, D. B. Rorabacher, Anal. Chem. 1999, 71, 3140-3144.

[14] F. Kaspar, 2021, DOI 10.5281/zenodo.5734257.

[15] V. L. Arcus, E. J. Prentice, J. K. Hobbs, A. J. Mulholland, M. W. Van der Kamp, C. R. Pudney, E. J. Parker, L. A. Schipper, Biochemistry 2016, 55, 1681-1688.

[16] A. Brand, L. Allen, M. Altman, M. Hlava, J. Scott, Learn. Publ. 2015, 28, 151-155.

[17] F. Crameri, G. E. Shephard, P. J. Heron, Nat. Commun. 2020, 11, 5444.

[18] C. Engler, R. Kandzia, S. Marillonnet, PLoS One 2008, 3, e3647

[19] S. Kanno, L. Cuyas, H. Javot, R. Bligny, E. Gout, T. Dartevelle, M. Hanchi, T. M. Nakanishi, M.-C. Thibaud, L. Nussaume, Plant Cell Physiol. 2016, 57, 690-706.

[20] A. A. Baykov, O. A. Evtushenko, S. M. Avaeva, Anal. Biochem. 1988, 171, 266-270.
[21] P. P. van Veldhoven, G. P. Mannaerts, Anal. Biochem. 1987, 161, 45-48.

[22] K. Itaya, M. Ui, Clin. Chim. Acta 1966, 14, 361366.

[23] S. D. Pegan, Y. Tian, V. Sershon, A. D. Mesecar, 2010, 13, 27-38.

[24] T. P. Geladopoulos, T. G. Sotiroudis, A. E. Evangelopoulos, Anal. Biochem. 1991, 192, 112116.

[25] R. F. Putnins, E. W. Yamada, Anal. Biochem. 1975, 68, 185-195.

[26] R. M. Flynn, M. E. Jones, F. Lipmann, J. Biol. Chem. 1954, 211, 791-796.

[27] C. H. Fiske, J. Subbarow, J. Biol. Chem. 1925.

[28] H. Goldenberg, A. Fernandez, Clin. Chem. 1966, 12, 871-882.

[29] T. Kuttner, H. R. Cohen, J. Biol. Chem. 1927.

[30] Z. He, C. W. Honeycutt, Commun. Soil Sci. Plant Anal. 2004, 36, 1373-1383.

[31] H. Katano, H. Watanabe, M. Takakuwa, C. Maruyama, Y. Hamano, Anal. Sci. 2013, 29, 10951098.

[32] E. S. Baginski, P. P. Foa, B. Zak, Clin. Chim. Acta 1967, 15, 155-158.

[33] D. T. Burns, D. Chimpalee, N. Chimpalee, S. Ittipornkul, Anal. Chim. Acta 1991, 254, 197-200.

[34] C. L. Penney, Anal. Biochem. 1976, 75, 201-210.

[35] N. Yoza, I. Akazaki, T. Nakazato, N. Ueda, H. Kodama, A. Tateda, Anal. Biochem. 1991, 199, 279-285.

[36] L. E. Guo, J. F. Zhang, X. Y. Liu, L. M. Zhang, H. L. Zhang, J. H. Chen, X. G. Xie, Y. Zhou, K. Luo, J. Yoon, Anal. Chem. 2015, 87, 1196-1201.

[37] M. A. Pesce, S. H. Bodourian, J. F. Nicholson, Clin. Chem. 1974, 20, 332-336.

[38] R. E. Reeves, L. K. Malin, Anal. Biochem. 1969, 28, 282-287.

[39] A. Ghetta, M. Matus-Ortega, J. García-Mena, G. Dehò, P. Tortora, M. E. Regonesi, Anal. Biochem. 2004, 327, 209-214.

[40] J. C. Johnson, M. Shanoff, S. T. Bass, J. A. Boezi, R. G. Hansen, Anal. Biochem. 1968, 26, 137-145.

[41] H. L. Drake, N. H. Goss, H. G. Wood, Anal. Biochem. 1979, 94, 117-120.

[42] D. R. Trentham, R. G. Bardsley, J. F. Eccleston, A G. Weeds, Biochem. J. 1972, 126, 635-644.

[43] R. W. Guynn, D. Veloso, R. L. Veech, Anal. Biochem. 1972, 45, 277-285.

[44] N. W. Cornell, M. G. Leadbetter, R. L. Veech, Anal. Biochem. 1979, 95, 524-526.

[45] D. W. Schulz, J. V Passonneau, O. H. Lowry, Anal. Biochem. 1967, 19, 300-314.

[46] A. S. G. Suárez, A. Stefan, S. Lemma, E. Conte, A. Hochkoeppler, Biotechniques 2012, 53, 99-103.

[47] Y. Machida, T. Nakanishi, Agric. Biol. Chem. 1982 , 46, 807-808.

[48] S. D. Haemmerli, Anal. Biochem. 1990, 1, 106109.

[49] H. Groot, T. Noll, Biochem. J. 1985, 230, 255-260.

[50] W. I. Hwang, S. Cha, Anal. Biochem. 1973, 55 379-387.

[51] P. Fossati, Anal. Biochem. 1985, 149, 62-65.

[52] A. Isobe, Y. Iwabuchi, M. Yajima, S.-I. Sakasegawa, Y. Yamaguchi, M. Seimiya, T. 
Umemura, S. Osawa, Clin. Chim. Acta. 2020, 511, 298-305.

[53] J. P. J. Ungerer, H. M. Oosthuizen, S. H. Bissbort, Clin. Chim. Acta 1993, 223, 148-157.

[54] M. Tagiri-Endo, Anal. Biochem. 2003, 315, 170 174.

[55] C. E. Rieger, J. Lee, J. L. Turnbull, Anal. Biochem. 1997, 246, 86-95.

[56] M. R. Webb, J. L. Hunter, Biochem. J. 1992, 287, 555-559.

[57] R. H. Upson, R. P. Haugland, M. N. Malekzadeh, R. P. Haugland, Anal. Biochem. 1996, 243, 41-45.

[58] U. Banik, S. Roy, Biochem. J. 1990, 266, 611-614.

[59] M. Hill, B. Arrio, Anal. Biochem. 1997, 254, 249253.

[60] C. Avila, B. A. Kornilayev, B. S. J. Blagg, Bioorg. Med. Chem. 2006, 14, 1134-1142.

[61] H. Nakamura, R. Yamazaki, T. Shirai, H. Sano, Y. Nakami, K. Ikebukuro, K. Yano, Y. Nomura, Y. Arikawa, Y. Hasebe, et al., Anal. Chim. Acta 2004, 518, 45-49.

[62] P. Mukherjee, S. Banerjee, A. Wheeler, L. A. Ratliff, S. Irigoyen, L. R. Garcia, S. W. Lockless, W. K. Versaw, Plant Physiol. 2015, 167, 628-638.

[63] T. Schenk, N. M. G. . Appels, D. A. van Elswijk, H. Irth, U. R. Tjaden, J. van der Greef, Anal. Biochem. 2003, 316, 118-126.

[64] C. Solscheid, S. Kunzelmann, C. T. Davis, J. L. Hunter, A. Nofer, M. R. Webb, Biochemistry 2015, 54, 5054-5062.

[65] P. Franz, V. Gassl, A. Topf, L. Eckelmann, B. Iorga, G. Tsiavaliaris, Biosens. Bioelectron. 2020, 169, 112616.

[66] R. Villamil-Ramos, A. K. Yatsimirsky, Chem. Commun. 2011, 47, 2694-2696.

[67] I. Debruyne, Anal. Biochem. 1983, 130, 454-460.

[68] J. K. Heinonen, S. H. Honkasalo, E. I. Kukko, Anal. Biochem. 1981, 117, 293-300.

[69] O. Hénin, B. Barbier, A. Brack, Anal. Biochem. 1999, 270, 181-184.

[70] S. Lenevich, M. D. Distefano, Anal. Biochem. 2011, 408, 316-320.

[71] R. K. Pathak, V. K. Hinge, A. Rai, D. Panda, C. P. Rao, Inorg. Chem. 2012, 51, 4994-5005.

[72] D. Chao, S. Ni, Sci. Rep. 2016, 6, 26477.

[73] S.-Y. Jiao, K. Li, X. Wang, Z. Huang, L. Pu, X.-Q. Yu, Analyst 2015, 140, 174-181.

[74] R. G. Hanshaw, S. M. Hilkert, H. Jiang, B. D. Smith, Tetrahedron Lett. 2004, 45, 8721-8724.

[75] X. Liu, H. T. Ngo, Z. Ge, S. J. Butler, K. A. Jolliffe, Chem. Sci. 2013, 4, 1680.

[76] B. K. Datta, S. Mukherjee, C. Kar, A. Ramesh, G. Das, Anal. Chem. 2013, 85, 8369-8375.

[77] Y. J. Jang, E. J. Jun, Y. J. Lee, Y. S. Kim, J. S. Kim, J. Yoon, J. Org. Chem. 2005, 70, 9603-9606.

[78] J. Wang, C.-S. Ha, Analyst 2010, 135, 1214-1218.

[79] K. K. Y. Yuen, K. A. Jolliffe, Chem. Commun. 2013, 49, 4824-4826.

[80] D. H. Lee, S. Y. Kim, J.-I. Hong, Angew. Chemie 2004, 116, 4881-4884.

[81] S. Anbu, S. Kamalraj, C. Jayabaskaran, P. S. Mukherjee, Inorg. Chem. 2013, 52, 8294-8296.

[82] K. M. Kim, D. J. Oh, K. H. Ahn, Chem. Asian J. 2011, 6, 122-127.
[83] J. H. Lee, A. R. Jeong, J.-H. Jung, C.-M. Park, J.-I. Hong, J. Org. Chem. 2011, 76, 417-423.

[84] V. Luxami, K. Paul, I. H. Jeong, Dalton Trans. 2013, 42, 3783-3786.

[85] P. Das, S. Bhattacharya, S. Mishra, A. Das, Chem. Commun. 2011, 47, 8118-8120.

[86] S. Goswami, A. K. Das, B. Pakhira, S. Basu Roy, A. K. Maity, P. Saha, S. Sarkar, Dalton Trans. 2014, 43, 12689-12697.

[87] G. Su, Z. Liu, Z. Xie, F. Qian, W. He, Z. Guo, Dalton Trans. 2009, 7888-7890.

[88] S. Bhowmik, B. N. Ghosh, V. Marjomäki, K. Rissanen, J. Am. Chem. Soc. 2014, 136, 55435546.

[89] N. Kumari, F. Zelder, Chem. Commun. 2015, 51, 17170-17173.

[90] J. Wen, Z. Geng, Y. Yin, Z. Zhang, Z. Wang, Dalt. Trans. 2011, 40, 1984-1989.

[91] H. J. Kim, J. H. Lee, J.-I. Hong, Tetrahedron Lett. 2011, 52, 4944-4946.

[92] I.-S. Shin, S. W. Bae, H. Kim, J.-I. Hong, Anal. Chem. 2010, 82, 8259-8265.

[93] H. N. Lee, K. M. K. Swamy, S. K. Kim, J.-Y. Kwon, Y. Kim, S.-J. Kim, Y. J. Yoon, J. Yoon, Org. Lett. 2007, 9, 243-246.

[94] M. Kumar, N. Kumar, V. Bhalla, Chem. Commun. 2013, 49, 877-879.

[95] M. J. McDonough, A. J. Reynolds, W. Y. G. Lee, K. A. Jolliffe, Chem. Commun. 2006, 2971-2973.

[96] H.-R. Xu, K. Li, M.-Q. Wang, B.-L. Wang, X. Wang, X.-Q. Yu, Org. Chem. Front. 2014, 1, 1276-1279.

[97] Z. Li, W. Zhang, X. Liu, C. Liu, M. Yu, L. Wei, RSC Adv. 2015, 5, 25229-25235.

[98] B. P. Morgan, S. He, R. C. Smith, Inorg. Chem. 2007, 46, 9262-9266.

[99] R. K. Pathak, K. Tabbasum, A. Rai, D. Panda, C. P. Rao, Anal. Chem. 2012, 84, 5117-5123.

[100] W.-H. Chen, Y. Xing, Y. Pang, Org. Lett. 2011, 13, 1362-1365.

[101] H. K. Cho, D. H. Lee, J.-I. Hong, Chem. Commun. 2005, 1690-1692.

[102] O. G. Tsay, S. T. Manjare, H. Kim, K. M. Lee, Y. S. Lee, D. G. Churchill, Inorg. Chem. 2013, 52, 10052-10061.

[103] B. Muthuraj, S. Mukherjee, S. R. Chowdhury, C. R. Patra, P. K. Iyer, Biosens. Bioelectron. 2017, 89, 636-644.

[104] X. Zhao, Y. Liu, K. S. Schanze, Chem. Commun. 2007, 2914-2916.

[105] J. F. Zhang, M. Park, W. X. Ren, Y. Kim, S. J. Kim, J. H. Jung, J. S. Kim, Chem. Commun. 2011, 47, 3568-3570.

[106] W. Zhu, X. Huang, Z. Guo, X. Wu, H. Yu, H. Tian, Chem. Commun. (Camb). 2012, 48, 1784-1786.

[107] M. J. Kim, K. M. K. Swamy, K. M. Lee, A. R. Jagdale, Y. Kim, S.-J. Kim, K. H. Yoo, J. Yoon, Chem. Commun. 2009, 7215-7217.

[108] J. Hatai, S. Pal, S. Bandyopadhyay, Tetrahedron Lett. 2012, 53, 4357-4360.

[109] X. Feng, Y. An, Z. Yao, C. Li, G. Shi, ACS Appl. Mater. Interfaces 2012, 4, 614-618.

[110] W. Yu, J. Qiang, J. Yin, S. Kambam, F. Wang, Y. Wang, X. Chen, Org. Lett. 2014, 16, 2220-2223.

[111] T. Cheng, T. Wang, W. Zhu, X. Chen, Y. Yang, Y. Xu, X. Qian, Org. Lett. 2011, 13, 3656-3659. 
[112] S. Mizukami, T. Nagano, Y. Urano, A. Odani, K. Kikuchi, J. Am. Chem. Soc. 2002, 124, 3920-3925.

[113] C. Lim, M. An, H. Seo, J. H. Huh, A. Pandith, A. Helal, H.-S. Kim, Sensors Actuators B Chem. 2017, 241, 789-799.

[114] Z. Li, H. Li, C. Shi, W. Zhang, W. Zhou, L. Wei, M. Yu, Sensors Actuators B Chem. 2016, 226, 127134.

[115] A. K. Dwivedi, G. Saikia, P. K. Iyer, J. Mater. Chem. 2010, 21, 2502-2507.

[116] G. Zhang, B. Lu, Y. Wen, L. Lu, J. Xu, Sensors Actuators B Chem. 2012, 171-172, 786-794.

[117] C. R. Lohani, J.-M. Kim, S.-Y. Chung, J. Yoon, K.H. Lee, Analyst 2010, 135, 2079-2084.

[118] X. Su, C. Zhang, X. Xiao, A. Xu, Z. Xu, M. Zhao, Chem. Commun. 2013, 49, 798-800.

[119] S. K. Asthana, A. Kumar, Neeraj, Shweta, S. K. Hira, P. P. Manna, K. K. Upadhyay, Inorg. Chem. 2017, 56, 3315-3323.

[120] D. H. Vance, A. W. Czarnik, J. Am. Chem. Soc. 1994, 116, 9397-9398.

[121] I.-B. Kim, M. H. Han, R. L. Phillips, B. Samanta, V. M. Rotello, Z. J. Zhang, U. H. F. Bunz, Chemistry 2009, 15, 449-456.

[122] S. K. Kim, N. J. Singh, J. Kwon, I.-C. Hwang, S. J. Park, K. S. Kim, J. Yoon, Tetrahedron 2006, 62, 6065-6072.

[123] J. Yoon, S. K. Kim, N. J. Singh, J. W. Lee, Y. J. Yang, K. Chellappan, K. S. Kim, J. Org. Chem. 2004, 69, 581-583.

[124] S. K. Kim, N. J. Singh, S. J. Kim, H. G. Kim, J. K. Kim, J. W. Lee, K. S. Kim, J. Yoon, Org. Lett. 2003, 5, 2083-2086.

[125] H.-R. Xu, K. Li, S.-Y. Jiao, S.-L. Pan, J.-R. Zeng, X.-Q. Yu, Analyst 2015, 140, 4182-4188.

[126] K. Ghosh, A. R. Sarkar, A. Samadder, A. R. KhudaBukhsh, Org. Lett. 2012, 14, 4314-4317.

[127] J.-H. Liao, C.-T. Chen, J.-M. Fang, Org. Lett. 2002 , 4, 561-564.

[128] P. Anzenbacher, K. Jursíková, J. L. Sessler, J. Am. Chem. Soc. 2000, 122, 9350-9351.

[129] D. Aldakov, J. P. Anzenbacher, Chem. Commun. 2003, 1394.

[130] P. Sokkalingam, D. S. Kim, H. Hwang, J. L. Sessler, C.-H. Lee, Chem. Sci. 2012, 3, 1819.

[131] X. Zhu, J. Yang, K. S. Schanze, Photochem. Photobiol. Sci. 2014, 13, 293-300.

[132] G. Sanchez, A. Espinosa, D. Curiel, A. Tarraga, P. Molina, J. Org. Chem. 2013, 78, 9725-9737.

[133] G. Sánchez, D. Curiel, W. Tatkiewcz, I. Ratera, A. Tárraga, J. Veciana, P. Molina, Chem. Sci. 2014, 5, 2328-2335.

[134] T. Gunnlaugsson, A. P. Davis, J. E. O'Brien, M. Glynn, Org. Lett. 2002, 4, 2449-2452.

[135] C. Caltagirone, C. Bazzicalupi, F. Isaia, M. E. Light, V. Lippolis, R. Montis, S. Murgia, M. Olivari, G. Picci, Org. Biomol. Chem. 2013, 11, 2445-2451.

[136] Y. Chen, W. Li, Y. Wang, X. Yang, J. Chen, Y. Jiang, C. Yu, Q. Lin, J. Mater. Chem. C 2014, 2, 4080.

[137] V. Jansson, K. Jansson, Anal. Biochem. 2003, 317, 268-270.

[138] G. A. Cook, W. E. O'Brien, H. G. Wood, M. Todd King, R. L. Veech, Anal. Biochem. 1978, 91, 557565.
[139] H. Flodgaard, Eur. J. Biochem. 1970, 15, 273-279.

[140] G. Lust, J. E. Seegmiller, Clin. Chim. Acta 1976, 66, 241-249.

[141] S. M. Marques, F. Peralta, J. C. G. Esteves da Silva, Talanta 2009, 77, 1497-1503.

[142] V. Jansson, K. Jansson, Anal. Biochem. 2002, 304, 135-137.

[143] P. Nyrén, Anal. Biochem. 1987, 167, 235-238.

[144] M. Ronaghi, S. Karamohamed, B. Pettersson, M. Uhlén, P. Nyrén, Anal. Biochem. 1996, 242, 84-89.

[145] Y. Sun, K. B. Jacobson, V. Golovlev, Anal. Biochem. 2007, 367, 201-209.

[146] B. A. Barshop, D. T. Adamson, D. C. Vellom, F. Rosen, B. L. Epstein, J. E. Seegmiller, Anal. Biochem. 1991, 197, 266-272.

[147] W. Xu, C. Shao, J. Pang, Y. Jiang, Y. Han, J. Wang, Electrochim. Acta 2019, 300, 171-176.

[148] G. Cohn, I. M. Kolthoff, Ind. Eng. Chem. Anal. Ed. 1942, 14, 886-890.

[149] M. Zarepour, K. Kaspari, S. Stagge, R. Rethmeier R. R. Mendel, F. Bittner, Plant Mol. Biol. 2010, 72, 301-310.

[150] S. Leimkühler, R. Hodson, G. N. George, K. V Rajagopalan, J. Biol. Chem. 2003, 278, 2080220811.

[151] C. Chen, G. Cheng, H. Hao, M. Dai, X. Wang, L. Huang, Z. Liu, Z. Yuan, PLoS One 2013, 8, e73912.

[152] C.-H. Wang, T.-X. Zhao, M. Li, C. Zhang, X.-H. Xing, Biotechnol. Lett. 2016, 38, 337-344.

[153] S. Beyaztaş, O. Arslan, J. Enzyme Inhib. Med. Chem. 2015, 30, 442-447.

[154] M. R. Rashidi, M. H. Soruraddin, F. Taherzadeh, A. Jouyban, Biochem. 2009, 74, 97-101.

[155] M. S. Drenichev, C. S. Alexeev, N. N. Kurochkin, S. N. Mikhailov, Adv. Synth. Catal. 2018, 360, 305312.

[156] K. F. Hellendahl, F. Kaspar, X. Zhou, Z. Yang, Z. Huang, P. Neubauer, A. Kurreck, ChemBioChem 2021, 22, 2002-2009.

[157] H. Yehia, S. Westarp, V. Röhrs, F. Kaspar, T. R. Giessmann, F. T. H. Klare, K. Paulick, P. Neubauer, J. Kurreck, A. Wagner, Molecules 2020, $25,934$.

[158] X. Zhou, K. Szeker, B. Janocha, T. Böhme, D. Albrecht, I. A. Mikhailopulo, P. Neubauer, FEBS J. 2013, 280, 1475-1490.

[159] K. Szeker, X. Zhou, T. Schwab, A. Casanueva, D. Cowan, I. A. Mikhailopulo, P. Neubauer, J. Mol. Catal. B Enzym. 2012, 84, 27-34.

[160] X. Zhou, W. Yan, C. Zhang, Z. Yang, P. Neubauer, I. A. Mikhailopulo, Z. Huang, Catal. Commun. 2019, 121, 32-37.

[161] S. Kamel, M. Weiß, H. F. T. Klare, I. A. Mikhailopulo, P. Neubauer, A. Wagner, Mol. Catal. 2018, 458, 52-59.

[162] S. Kamel, C. Walczak, P. Neubauer, A. Wagner, 2020, in preparation.

[163] X. Zhou, K. Szeker, L.-Y. Jiao, M. Oestreich, I. A. Mikhailopulo, P. Neubauer, Adv. Synth. Catal. 2015, 357, 1237-1244.

[164] F. Kaspar, P. Neubauer, A. Kurreck, ChemBioChem 2020, 22, 1385-1390.

[165] Y. H. Jang, L. C. Sowers, T. Çağin, W. A. Goddard, J. Phys. Chem. A 2001, 105, 274-280. 
[166] J. Reijenga, A. van Hoof, A. van Loon, B. Teunissen, Anal. Chem. Insights 2013, 8, ACI.S12304.

[167] R. T. Giessmann, N. Krausch, F. Kaspar, N. M. Cruz Bournazou, A. Wagner, P. Neubauer, M. Gimpel, Processes 2019, 7, 380.

[168] D. Salavera, S. K. Chaudhari, X. Esteve, A. Coronas, J. Chem. Eng. Data 2005, 50, 471-476.

[169] M. J. Pugmire, S. E. Ealick, Biochem. J. 2002, 361, $1-25$.

[170] J. M. Davies, R. J. Poole, D. Sanders, Biochim. Biophys. Acta - Bioenerg. 1993, 1141, 29-36.

[171] A. Hachimori, A. Takeda, M. Kaibuchi, N. Ohkawara, T. Samejima, J. Biochem. 1975, 77, 1177-1183.

[172] S. M. Lambert, J. I. Watters, J. Am. Chem. Soc. 1957, 79, 5606-5608.

[173] T. A. Wright, J. M. Stewart, R. C. Page, D. Konkolewicz, J. Phys. Chem. Lett. 2017, 8, 553558.

[174] E. Gasteiger, C. Hoogland, A. Gattiker, S. Duvaud, M. R. Wilkons, R. D. Appel, A. Bairoch, in Proteomics Protoc. Handb. (Ed.: J. Walker), Humana Press, 2005, pp. 571-607.

[175] J. K. Hobbs, W. Jiao, A. D. Easter, E. J. Parker, L. A. Schipper, V. L. Arcus, ACS Chem. Biol. 2017, $12,868$.

[176] J. K. Hobbs, W. Jiao, A. D. Easter, E. J. Parker, L. A. Schipper, V. L. Arcus, ACS Chem. Biol. 2013, 8, 2388-2393.

[177] V. L. Arcus, A. J. Mulholland, Annu. Rev. Biophys. 2020, 49, 163-180.

[178] H. Eyring, J. Chem. Phys. 1935, 3, 107-115.

[179] H. Akaike, in 2nd Int. Symp. Inf. Theory, Tsahkadsor, Armen. USSR (Eds.: B.N. Petrov, F. Csáki), 1973, pp. 267-281.

[180] S. A. Hindson, H. A. Bunzel, B. Frank, D. A. Svistunenko, C. Williams, M. W. van der Kamp, A. J. Mulholland, C. R. Pudney, J. L. R. Anderson, ACS Catal. 2021, 11, 11532-11541.

[181] H. A. Bunzel, H. Kries, L. Marchetti, C. Zeymer, P. R. E. Mittl, A. J. Mulholland, D. Hilvert, J. Am. Chem. Soc. 2019, 141, 11745-11748.

[182] J. K. Hobbs, C. Shepherd, D. J. Saul, N. J. Demetras, S. Haaning, C. R. Monk, R. M. Daniel, V. L. Arcus, Mol. Biol. Evol. 2012, 29, 825-835.

[183] M. W. van der Kamp, E. J. Prentice, K. L. Kraakman, M. Connolly, A. J. Mulholland, V. L. Arcus, Nat. Commun. 2018, 9, 1177.

[184] R. S. Firestone, S. A. Cameron, J. M. Karp, V. L. Arcus, V. L. Schramm, ACS Chem. Biol. 2017, 12, 464-473.

[185] M. E. Peterson, R. M. Daniel, M. J. Danson, R. Eisenthal, Biochem. J. 2007, 402, 331-337.

[186] M. E. Peterson, R. Eisenthal, M. J. Danson, A. Spence, R. M. Daniel, J. Biol. Chem. 2004, 279, 20717-20722.

[187] R. S. Phillips, S. Craig, A. Kovalevsky, O. Gerlits, K. Weiss, A. I. lorgu, D. J. Heyes, S. Hay, ACS Catal. 2020, 10, 1692-1703.

[188] H. B. L. Jones, R. M. Crean, A. Mullen, E. G. Kendrick, S. D. Bull, S. A. Wells, D. R. Carbery, F. MacMillan, M. W. van der Kamp, C. R. Pudney, Biochemistry 2019, 58, 2362-2372.

[189] H. B. L. Jones, R. M. Crean, C. Matthews, A. B. Troya, M. J. Danson, S. D. Bull, V. L. Arcus, M. W. van der Kamp, C. R. Pudney, ACS Catal. 2018, 8, 5340-5349.

[190] E. J. Prentice, J. Hicks, H. Ballerstedt, L. M. Blank, L. L. Liáng, L. A. Schipper, V. L. Arcus, Biochemistry 2020, 59, 3562-3569.

[191] F. Kaspar, P. Neubauer, A. Kurreck, ChemPhysChem 2021, 22, 283-287.

[192] R. G. Silva, M. J. Vetticatt, E. F. Merino, M. B. Cassera, V. L. Schramm, J. Am. Chem. Soc. 2011, 133, 9923-9931. 